\title{
Challenges for stellar pulsation and evolution theory
}

\author{
J. Daszyńska-Daszkiewicz \\ Instytut Astronomiczny, Uniwersytet Wrocławski, Kopernika 11, 51-622 Wrocław, Poland
}

\begin{abstract}
During the last few decades, great effort has been made towards understanding hydrodynamical processes which determine the structure and evolution of stars. Up to now, the most stringent constraints have been provided by helioseismology and stellar cluster studies. However, the contribution of asteroseismology becomes more and more important, giving us an opportunity to probe the interiors and atmospheres of very different stellar objects. A variety of pulsating variables allows us to test various parameters of micro- and macrophysics by means of oscillation data. I will review the most outstanding key problems, both observational and theoretical, of which our knowledge can be improved by means of asteroseismology.
\end{abstract}

\section{Introduction}

Stars are the main components of the visible Universe. Our understanding of their internal structure and the way they evolve is a crucial piece in understanding how galaxies form and evolve. Studies of stellar clusters bring general information about the composition and evolution of stars but they are not sensitive enough to teach us about microphysical processes determining stellar structure. The only particles that carry information about the solar centre are neutrinos, which are a direct byproduct of nuclear fusion reactions. As a detection of these weakly interacting particles is very difficult, and for stars other than the Sun still beyond our reach, pulsations provide the only opportunity for testing the physics of stellar interiors and, in a next step, theory of stellar evolution.

Helioseismology has by far the greatest contribution to theory of stellar structure and evolution. The global studies led to determinations of the solar age, the depth of the convective zone, helium abundance and rotational profile. The great impact of helioseismology concerns also atomic physics, exemplified by its role in solving the solar neutrino problem, or by testing opacity data and equation of state. Now a new era is opening up for local helioseismology, which should provide three-dimensional maps of the solar interior and magnetic field.

Pulsating variables cover a wide range of masses and every stage in stellar evolution. As a consequence we can observe pulsations with various periods, amplitudes and shapes of light curves which result from excitation of different modes. In spite of this variety, there are only two underlying mechanisms for driving stellar pulsations. The first is self excitation in the layers which operate as a heat engine. This instability mechanism excites pulsations in most stars, beginning from classical instability strip stars, through B type main sequence stars, hot subdwarfs to white dwarfs. The second way to make a star pulsate is to force stochastic oscillations by turbulent convection. This stochastic excitation drives solar-like oscillations, including those observed in the Sun, and is expected in all stars with extended convective outer layers. The most complete version of the Hertzsprung-Russell diagram of pulsating 
stars is shown in Fig. 1. This diagram was constructed by Simon Jeffery on the basis of an idea by Jørgen Christensen-Dalsgaard.

The diversity of stellar pulsations offers an opportunity to probe various physical phenomena, like element mixing, opacity, efficiency of convection, magnetic field and non-uniform rotation. The ultimate goal of asteroseismology is to construct a seismic model which reproduces all observed frequencies and the corresponding pulsational mode characteristics. During the last few years we are witnessing a growing impact of asteroseismology in extracting constraints on stellar parameters and physical processes in the stellar interiors. Another door to the application of asteroseismology is opening by pulsating stars which harbour planets, like $\mu$ Ara, a solar-like star (Bazot et al. 2005) or V391 Peg, a hot subdwarf (Silvotti et al. 2007).

In the first section, I will list observational key problems, which I consider most challenging for theory of stellar evolution and pulsation. The second section is devoted to the most puzzling theoretical aspects. Conclusions end this review. Because of the space limit, I had to make a crude selection. Therefore, I did not discuss, for example, the problems connected with the presence of magnetic field and the potential of asteroseismology to test it. These issues can be found, e.g., in a review by Kochukhov (2009) of the roAp pulsators.

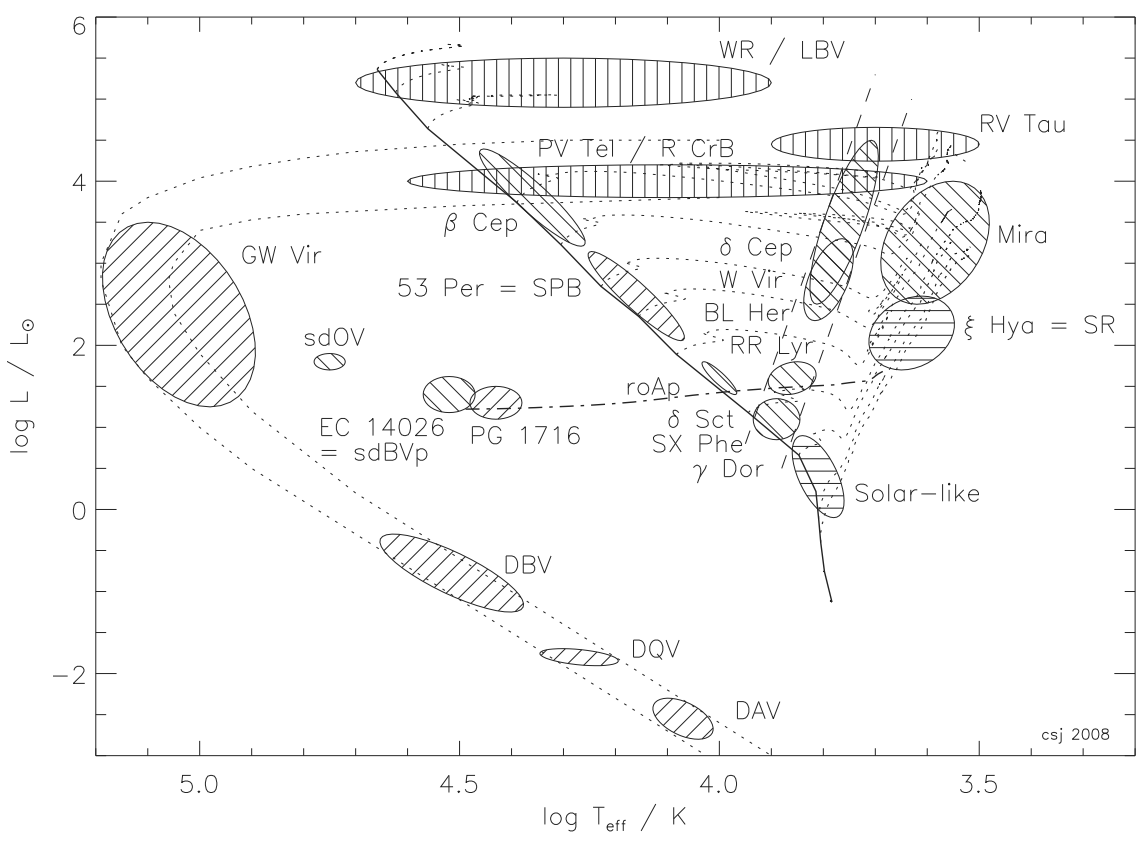

Figure 1: The Hertzsprung-Russell diagram with schematic locations of various types of pulsating variables. The hatching styles correspond to different types of stellar pulsation: diagonal lines - heat-driven $\mathrm{p}$ - and g-mode pulsations, horizontal lines - solar-like oscillations, and vertical lines - strange mode (highly non-adiabatic) pulsations. The zero age main sequence is depicted by a heavy solid line and the zero age horizontal branch by a heavy dot-dash line. Evolutionary tracks and white dwarf cooling sequences are shown as dotted lines. Figure courtesy of Simon Jeffery (2008a). 


\section{Observational challenges}

\section{Classical Cepheids}

Classical Cepheids play a crucial role in astronomy and their importance has been recognized a long time ago. Firstly, they are primary distance indicators on the extragalactic scale thanks to the Period Luminosity Relation discovered by Henrietta Leavitt in 1908. Secondly, their pulsations provide a test of stellar evolution theory of intermediate mass stars.

The longest standing problem connected with these variables was a discrepancy for doublemode Cepheids between masses estimated from pulsation and evolution theory. The implementation of new opacity data (Iglesias \& Rogers 1991) made by Moskalik et al. (1992) allowed essentially to reconcile this mass discrepancy. Despite this crucial work some disagreement still remains and possible sources of this persistent problem are opacities, mass loss and internal mixing processes (Keller 2008 and reference therein). Recently, yet another problem arose for double-mode Cepheid models. Smolec \& Moskalik (2008a, b) have shown that if buoyancy forces in convectively stable layers are included in pulsation modelling, double-mode solutions do not exist.

During the last few years a number of interesting and challenging discoveries were made, based mostly on the OGLE project data. There are:

- Blazhko Cepheids (Moskalik \& Kołaczkowski 2008a)

- non-radial modes in classical Cepheids (Moskalik \& Kołaczkowski 2008b)

- 10/30 double-mode Cepheids (Soszyński et al. 2008a)

- single-mode second-overtone Cepheids (Udalski et al. 1999, Soszyński et al. 2008b),

- triple-mode Cepheids (Moskalik et al. 2004, Soszyński et al. 2008a, b)

- eclipsing binary systems containing Cepheids (Soszyński et al. 2008b).

All the above theoretical and observational facts wait for explanation and call for new generation models of stellar pulsation and evolution.

\section{B type main sequence stars}

Studying B type main sequence stars is of great importance because those with masses greater than about $8 \mathrm{M}_{\odot}$ are progenitors of Type II Supernovae, whereas those with masses less than about $8 \mathrm{M} \odot$ form the CNO elements in the Universe. In some of these objects pulsation occurs, giving an opportunity to study physics of stellar interiors by means of oscillation data. There are two classes of $B$ type main sequence pulsators: 1) $\beta$ Cephei stars with masses larger than $8 \mathrm{M}_{\odot}$ and spectral types $\mathrm{B} 0-\mathrm{B} 2.5$, in which mainly pressure $(\mathrm{p})$ modes are excited, and 2) Slowly Pulsating $B$ type $(S P B)$ stars with masses smaller than $8 \mathrm{M}_{\odot}$ and spectral types B3-B9, which pulsate in high-order gravity $(\mathrm{g})$ modes.

Many years had elapsed since the discovery of $\beta$ Cephei stars before the cause of their pulsations was identified. As in the case of the classical Cepheid mass discrepancy problem, the reason was that the Los Alamos opacities missed the metal bump because of underestimations of the heavy element contributions, mostly from the iron group (e.g., Moskalik \& Dziembowski 1992, Dziembowski et al. 1993). As pulsations in $\beta$ Cephei and SPB stars are strictly connected with the metal opacity bump, nobody expected to find many of them in the Magellanic Clouds (MCs), because the metallicity in the MCs is much lower than in the Milky Way; $Z=0.007$ for the Large Magellanic Cloud and $Z=0.002$ for the Small Magellanic Cloud. Therefore, the discovery of a large number of $\beta$ Cep and SPB variables in the MCs was rather a surprise. This started with the pioneering paper by Pigulski and Kołaczkowski (2002). The later papers by Kołaczkowski et al. (2006) and Karoff et al. (2008) increased the number of known B type main sequence pulsators in the MCs. Moreover, the newly determined solar chemical composition (Asplund et al. 2005, AGS05) gives a lower metallicity of about $Z=0.012$ compared to the older one (Grevesse \& Noels 1993) $Z=0.017$. Nevertheless, the new $Z$ value does not cause disappearance of the $B$ main sequence instability 
strip, as one would initially expect, because most of the reduction was obtained in the CNO elements and now the relative abundances of Fe group elements are significantly higher than the older ones (Pamyatnykh \& Ziomek 2007, Miglio et al. 2007). However, the problem with the existence of $\beta$ Cep stars in SMC remains open. This observational fact waits for an explanation and indicates that improvements are still needed in the treatment of opacities, mixing processes, diffusion etc., as well as more observational data should be gathered.

Another challenge is the occurrence of hybrid B type pulsators like $\nu$ Eri and $12 \mathrm{Lac}$ (e.g., see Handler 2009). The problem is that the lower frequencies observed in the g mode range cannot reach the instability in pulsational models. This instability problem occurs also for very high-frequency modes. These two stars were recently studied by Dziembowski \& Pamyatnykh (2008), who highlighted problems of mode excitation, uncertainties in opacity data and element distribution, extent of the overshooting distance and internal rotation.

\section{Hot subdwarfs}

B type subdwarfs are stars in the core helium burning phase with a thin hydrogen envelope. No hydrogen shell burning takes place. These stars represent the final stage before the white dwarf phase but only $2 \%$ of white dwarfs are formed through this channel.

Short period pulsations in these objects were theoretically predicted by Charpinet et al. (1997); Kilkenny et al. (1997) found them observationally. Then, Green et al. (2003) found $B$ type subdwarfs pulsating with long periods. Both types of pulsation are driven by the $\kappa$ mechanism due to the Z-opacity bump. Charpinet et al. (1997) incorporated a nonuniform iron profile as determined by the condition of diffusive equilibrium between gravitational settling and radiative levitation. This allowed removing the artificial assumption of a very high value of the metallicity. The present status of the hot subdwarfs asteroseismology was summarized by Fontaine et al. (2008a). The evolutionary status of subluminous B type stars seems to be proven but not their origin. There are two most supported scenarios: 1) single star evolution, or 2) binary star evolution, which can be a common envelope evolution, a stable Roche lobe overflow or a merger of two He WD stars.

Recently, also pulsation in O type subdwarfs was discovered (Woudt et al. 2006, RodriguezLopez et al. 2007). The sdO stars are the more evolved and even more puzzling cousins of sdB stars. These objects have a $\mathrm{C} / \mathrm{O}$ core and are in the helium burning shell phase. As they cover quite a large part of the HR diagram, they are thought to have many different origins. The "luminous" (low gravity) sdO stars are believed to be post-AGB stars. For the "compact" (high gravity) sdO stars two origin scenarios were proposed depending on the helium abundance: 1) post EHB objects (descendants of sdBs) for He-deficient sdOs, and 2) a merger of two He WDs or a delayed core He flash scenario for He-enriched sdOs (Rodriguez-Lopez et al. 2007). Fontaine et al. (2008a) performed non-adiabatic pulsational calculations taking into account time-dependent diffusion, as has been done before for sdB pulsators. They showed that radiative levitation causes pulsational instabilities in the sdO stars through an iron accumulation in the driving region.

There is great potential for asteroseismology of OB subdwarf pulsators because their radiative atmospheres combined with high gravities make them ideal for investigating diffusive processes. The problem with each origin scenario of sdOB stars is that a large amount of hydrogen has to be lost before or just at the beginning of helium core ignition. As in the case of the main sequence $B$ type pulsators, additional opportunities come from hybrid sdOB pulsators (Schuh et al. 2006, Lutz et al. 2008).

\section{Extreme helium stars}

The extreme helium stars are low-mass highly evolved objects (supergiants) of B and A types, with spectra showing very weak or no hydrogen lines (Jeffery 2008b). They cover a wide range 
of effective temperature and most of them have luminosities close to the Eddington limit. The fundamental questions about this kind of stars are their origin (how to remove a hydrogenrich envelope) and their connection with stars with normal He abundance. Two main origin scenarios were proposed. In the first scenario, the EHe star is a direct product of a single white dwarf which underwent a late thermal pulse (e.g., Iben et al. 1983). The second scenario involves a white dwarf binary which has merged (e.g., Webbink 1984).

The existence of EHe stars exhibiting pulsational variability is extremely significant. Jeffery (2008c) introduced consistent classification of variable EHe stars. The first group consists of PV Tel stars with strange mode pulsational instability (Saio \& Jeffery 1988) which is supposed to be present in all stars with sufficiently high luminosity/mass ratio. The second class includes $\mathrm{BX}$ Cir stars with pulsations driven by the $\kappa$ mechanism operating in the $\mathrm{Z}$ bump (Saio 1993). Here, the role of the iron group element opacity is increased due to a reduction of hydrogen (Jeffery \& Saio 1999).

There are also helium-rich subluminous OB stars whose origin is unclear (Napiwotzki 2008, Ahmad \& Jeffery 2008), but the hypothesis of merging two He core white dwarfs seems to be preferred. Questions arise about their connection with hydrogen-rich sdOB stars or with EHe stars. In the light curve of one He sdB object, Ahmad \& Jeffery (2005) detected multiperiodic variations. These authors have shown that the variations can be associated with high-order g-mode pulsations, but according to theory such modes are stable.

New class of white dwarfs

Asteroseismology of white dwarf (WD) pulsators provides many constraints on stellar physics and evolution. For example, from period changes we can estimate the cooling rate, which in turn can give information on the age of our Galaxy and can measure the neutrino generation rate in hot WDs (Winget 1998). WD asteroseismology also supplies a unique test of the equation of state of matter at high densities and temperatures. Because most stars (97\%) will end up as WDs, their studies are of special importance. An excellent review on the three types of WD pulsators, i.e., ZZ Cet (DAV), V777 Her (DBV) and GW Vir (PNNV+DOV) type stars, can be found in Fontaine \& Brassard (2008).

WDs of DA spectral type have hydrogen-rich atmospheres. The atmospheres of the two other types (DB, DO+PNNV) are dominated by helium. There is one more class of WDs at $T_{\text {eff }} 11000-13000 \mathrm{~K}$, with carbon lines in their spectra, designated the DQ type, but until recently even in this class, helium was believed to be the dominant component of the atmosphere. Therefore, the discovery of hot DQ WDs $\left(T_{\text {eff }} 18000-23000 \mathrm{~K}\right)$ with carbondominated atmospheres and little or no $\mathrm{H}$ and $\mathrm{He}$, was rather unexpected (Dufour et al. 2007). The origin of the cooler DQ WDs is quite well understood in the framework of the model of carbon dredge-up by the deep helium convection zone. On the other hand, the origin of hot DQ WDs cannot be explained within any known post-AGB evolution channel.

Soon thereafter, Fontaine et al. (2008b) have explored the instability of hot DQ WD models against pulsation. Their analysis showed that gravity modes should be excited in such models in the period range $100-700 \mathrm{~s}$. Almost at the same time, Montgomery et al. (2008) announced the photometric variability of one carbon-atmosphere object with a period of $417 \mathrm{~s}$. This makes this object a prototype of a new class of pulsating WDs. Recently, two more variable DQ WDs have been discovered by Barlow et al. (2008). Having in mind the potential of asteroseismology, one can expect interesting results from seismic studies of hot DQ WD pulsators before long. 


\section{Theoretical challenges}

\section{Opacities and chemical composition}

Stellar opacities constitute a vital component in modelling stellar structure because they determine the transport of radiation through matter. Their values depend on the temperature, density and chemical composition, $\kappa\left(T, \rho, X_{i}\right)$. For a long time the only source of the opacity data for astrophysical purposes was the Los Alamos Opacity Library (LAOL). The LAOL was in use until 1990, although already in 1982 N. R. Simon dared to blame them for the failure in explaining the Cepheid mass discrepancy and pulsations of $\beta$ Cephei stars, and urged reexamination of this crucial input (Simon 1982). Finally, in early 90ties, two teams of atomic physicists recomputed the opacity data and found that the LAOL underestimated the contributions from the heavy elements by a factor of $2-3$ at a temperature of about $200000 \mathrm{~K}$. The first team was represented by Iglesias \& Rogers (e.g., 1991, 1996), who called their results OPAL (OPAcity Library). The second was an international team led by M. J. Seaton and their opacities were named OP (Opacity Project, e.g., Seaton 1992, 1996, 2007).

The computation of new opacity tables was a milestone for astrophysics and the main consequences of a huge enhancement of the metal opacity bump were: 1) the seismic model of the Sun was improved, 2) the Cepheid mass discrepancy was significantly reduced, 3) the pulsations of $B$ type main sequence stars and of 4) some extreme He stars were explained, 5) pulsations of sdB and sdO stars were predicted.

The opacity data are being constantly updated but the main features are kept unchanged. Recently, a substantial revision of the solar chemical mixture has led to a significant change of the opacity values (AGS05). The new solar composition was reduced mostly in the CNO elements and now the solar metallicity is only $70 \%$ of the older one by Grevesse \& Noels (1993). As mentioned before, these new solar abundances did not diminish the extent of the pulsational instability strip of B type stars because the relative Fe abundance was increased, hence the role of the $\mathrm{Z}$ bump opacity in driving pulsations was amplified. There is other strong evidence supporting the AGS05 mixture. For example, now the solar metallicity is in better agreement with metallicities of stars in its neighbourhood. Then, galactic beat Cepheid models with the AGS05 abundances better fit the observations (Buchler \& Szabo 2007). Moreover, with reduced $Z$, the pre-main sequence models show smaller lithium depletion (Montalbán \& D'Antona 2006) which brings them closer to the observations. However, the new solar composition had bad consequences for the helioseismic model which lost consistency with the standard solar model. This problem was extensively discussed in a review by Basu \& Antia (2008)

There are many papers in which stellar pulsations of various objects were used as a test of opacity data, e.g., Dziembowski \& Pamyatnykh (2008) for $\beta$ Cep stars, Jeffery \& Saio (2006) for pulsating subdwarf B stars, Lenz et al. (2008) for $\delta$ Sct stars or Théado et al. (2009) for roAp stars. The role of input from opacity data in pulsation computations was recently summed up by Montalbán \& Miglio (2008).

\section{Rotation}

A credible theory of stellar structure and evolution should incorporate rotation. Firstly, rotation affects the stellar structure by breaking spherical symmetry. The most extreme example known is Achernar with a ratio of the major to minor axes equal to $1.56 \pm 0.05$. Secondly, rotation activates various processes, like meridional circulation, shear instabilities, diffusion, horizontal turbulence, which cause mixing. Thirdly, the distribution of internal angular momentum is determined through different rotational velocity at different depths. And finally, mass loss from the surface can be enhanced by rapid rotation through centrifugal effects. Many excellent papers on stellar rotation have been published by, e.g., Sweet, Öpik, Tassoul, Roxburgh, Zahn, Spruit, Deupree, Talon, Meynet, Maeder, Mathis, but there is no space here 
to mention the results of all of them. In the last years the most comprehensive studies of the rotational effects on stellar structure and evolution were presented in a series of papers by Maeder, Meynet and their collaborators (e.g., Maeder \& Meynet 2000, Meynet \& Maeder 2000).

How rotation affects pulsation depends on the rotation rate and on the closeness of oscillation frequencies. If the pulsational frequency is much larger than the rotational angular velocity, the perturbation approach is applicable; this considerably simplifies calculations and in the first-order approximation each pulsational mode can be described by a single spherical harmonic. This is no longer true if the higher-order effects of rotation are included (Dziembowski \& Goode 1992). Moderate rotation can also couple modes for which the frequency distance is close to the rotational angular velocity and where the spherical harmonic indices satisfy the relations: $l_{j}=l_{k}+2$ and $m_{j}=m_{k}$ (Soufi et al. 1998). The third-order expression for a rotationally split frequency can by found in Goupil et al. (2000). As for mode geometry, the main effect of fast rotation is the confinement of pulsation towards the stellar equator (e.g., Townsend 1997).

An entirely different approach has to be applied if the pulsation frequencies are of the order of or smaller than the rotation frequency $\left(\nu_{\text {puls }} \sim \nu_{\text {rot }}\right)$. Three different treatments of slow modes can be found in the literature: the traditional approximation (Townsend 2003), expansion in Legendre function series (Lee \& Saio 1997) and 2D $(r, \theta)$ modelling (Savonije 2007).

Rotation also complicates the identification of pulsation modes from photometric diagnostic diagrams because they become dependent on inclination angle, $i$, azimuthal order, $m$, and rotation velocity, $v_{\text {rot }}$. This problem was studied by Daszyńska-Daszkiewicz et al. (2002) for coupled modes, and by Townsend (2003) and Daszyńska-Daszkiewicz et al. (2007) for slow modes.

The asteroseismic potential of rotating pulsators lies in the rotational splitting kernel, $K(r)$ that gives information on the rotational profile $\Omega(r)$. The result that the rotation rate increases inward was obtained from studies of many pulsating stars, e.g., Goupil et al. (1993) for the $\delta$ Sct star GX Peg; Dziembowski \& Jerzykiewicz (1996) for the $\beta$ Cep star 16 Lac; Aerts et al. (2003) for the $\beta$ Cep star V836 Cen, Pamyatnykh et al. (2004) for the $\beta$ Cep star $\nu$ Eri and Dziembowski \& Pamyatnykh (2008) for the $\beta$ Cep stars $\nu$ Eri and 12 Lac.

Another question is about the impact of pulsation on rotational evolution. Talon \& Charbonnel (2005) showed that internal gravity waves contribute to braking rotation in the inner regions of low-mass stars. Computations by Townsend \& MacDonald (2008) demonstrated that pulsation modes can redistribute angular momentum and trigger shear instability mixing in the $\mu$ gradient zone. Mathis et al. (2008) discussed the transport of angular momentum in the solar radiative zone by internal gravity waves.

\section{Convection}

Convection plays a very important role in the transport of energy and mixing of matter. It is a complex physical process with a three-dimensional, non-local and time-dependent character. The convective cells are also a source of acoustic waves in subphotospheric layers, leading to stochastic excitation of stellar oscillations. In turn, dissipation of acoustic energy heats stellar chromospheres, causing "fingerprints" in spectral lines. Also stellar activity is a result of joint action of convection and differential rotation.

The most widely used description of stellar convection is the Mixing Length Theory (MLT) due to Böhm-Vitense (1958) and some modifications of it. In the framework of MLT, the size of convective elements is parameterized by the the mixing length parameter, $\alpha_{\text {conv }}$, which is adjusted to fit some observational quantities. A step forward was done by Canuto et al. (1996), who formulated a theory of turbulent convection taking into account the full spectrum of convective eddies. A 3D hydrodynamical simulation by Stein \& Nordlund (1998) allowed 
to reproduce qualitatively convection in the solar surface layers.

A discussion of differences between the 3D hydrodynamical model of convection and 1D MLT model was presented by Steffen (2007). The main results of his comparisons were that it is impossible to reproduce the correct temperature profile with any value of the MLT parameter, and that the radiative layer between two convection zones is completely mixed.

The presence of pulsation in stars with expanded convective envelopes complicates the picture even more. This is the case for classical Cepheids, RR Lyrae stars, red giant pulsators, $\delta$ Scuti and $\gamma$ Doradus stars, as well as for pulsating white dwarfs of the V777 Her and ZZ Cet types. A simplistic approach to pulsational modelling of these objects is a convective flux freezing approximation which assumes that the convective flux is constant during the pulsation cycle. Although in some $\delta$ Scuti stars convection seems to be inefficient (DaszyńskaDaszkiewicz et al. 2003, 2005a), this is a crude approximation and an adequate treatment of pulsation-convection interaction should be applied.

The first formulation of pulsation-convection interactions in which convection is non-local and time-dependent was given by Unno (1967) and Gough (1977). Following this concept and its variants, many research groups undertook attempts to model stellar oscillations in various objects: solar-like stars (Houdek, Goupil, Samadi), $\delta$ Scuti and $\gamma$ Doradus stars (Xiong, Houdek, Dupret, Grigahcene, Moya), classical Cepheids and RR Lyr variables (Feuchtinger, Stellingwerf, Buchler, Kollath, Smolec, Moskalik), pulsating red giants (Xiong, Deng, Cheng) and recently, V777 Her (DBV) white dwarfs (Quirion, Dupret).

The potential of asteroseismology for estimating the size of the convective core in massive stars was also explored. Dziembowski \& Pamyatnykh (1991) demonstrated that modes which are largely trapped in the region surrounding the convective core boundary can measure the extent of overshooting. The first evidence of core overshooting in a $\beta$ Cep star was found by Aerts et al. (2003) for V836 Cen. Miglio et al. (2008) studied the sensitivity of highorder $\mathrm{g}$ modes in SPB and $\gamma$ Dor stars to the properties of convective cores. In particular, the period spacing of gravity modes depends on the location and shape of the chemical composition gradient.

In general, convective transport of energy above the core in OB type stars is negligible. However, recently Maeder et al. (2008) have shown that very fast rotation in massive OB stars can increase the extent of outer convective envelopes. This has many consequences, e.g., acoustic modes can be generated. Also, stars close to the Eddington limit may develop a convective envelope.

\section{Mass loss}

Mass loss occurs in all late evolutionary phases and in massive stars. There are two principal mechanisms driving stellar winds: radiation driving in hot stars and dust driving in cool and luminous stars (e.g., Owocki 2004). For most stars, no consistent description of the mass loss rate exists and empirical formulae are used instead. Also, the interaction between mass loss and rotation has not yet been fully understood (e.g., Maeder \& Meynet 2004, Owocki 2008). Some stars with strong winds exhibit also pulsations. These are Mira and semi-regular (SR) variables, massive OB main sequence stars, Wolf-Rayet stars and Luminous Blue Variables (LBV). For such objects a natural question emerges about pulsation and mass loss coupling. It has been recognized many years ago that pulsations in large amplitude variables, like Miras or Cepheids, can enhance mass loss (e.g., Wood 2007, Neilson \& Lester 2008). Constraints have been derived from relations between the mass loss rate and pulsational period and between the wind outflow speed and pulsational period (e.g., Knapp et al. 1998). As for hot pulsators, Howarth et al. (1993) found wind variability in $\zeta$ Oph with the pulsational cycle, and Kaufer et al. (2006) detected the pulsation beat period in $\mathrm{H} \alpha$ profile observations for a B0 type supergiant. A nice discussion of the coupling between mass loss and pulsation in massive stars can be found in Townsend (2007). 
Interesting results have been obtained recently by Quirion, Fontaine \& Brassard (2007) for hot white dwarf pulsators of the GW Vir type. The authors showed that useful constraints on mass loss can be inferred from the red edge position of the instability strip.

\section{Conclusions}

An ultimate goal of asteroseismology is to help solving the equation observation = theory and to avoid the equation more data = less understanding. But as we are all aware, a more realistic treatment of macro- and microphysics in stellar modelling would be desirable.

One should have in mind that not only pulsation frequencies can probe stellar structure. An ideal seismic stellar model should account both for all measured oscillation frequencies and for associated pulsation mode characteristics. Therefore more use should be made of photometric and spectroscopic observables, i.e., amplitudes and phases of photometric and spectroscopic variations, and simultaneous multi-colour photometric and spectroscopic observations should be carried out. From such data we can infer an additional asteroseismic quantity, which is the ratio of the bolometric flux variations to the radial displacement. This new asteroseismic probe, called the $f$ parameter, is determined in subphotospheric layers, and therefore it is complementary to the frequency data which poorly probe these stellar regions. The value of $f$ is very sensitive to global stellar parameters, element mixture (hence the mixing processes), opacities and subphotospheric convection. Therefore, a comparison of empirical and theoretical values of $f$ provides additional stringent constraints on various physical parameters and processes. Such asteroseismic studies were proposed by DaszynskaDaszkiewicz et al. $(2003,2005 \mathrm{ab})$ and successfully applied to $\delta$ Scuti and $\beta$ Cephei stars. In the case of $\delta$ Scuti stars, useful constraints on subphotospheric convection were derived, and in the case of $\beta$ Cep stars, on opacities.

In the next step, the $f$ parameter should be fitted to observations together with the pulsation frequency. These complex asteroseismic studies should lead to stronger constraints, improving our knowledge in theory of stellar pulsation and evolution.

Acknowledgments. The author would like to thank the SOC members of the JENAM 2008 Symposium No. 4 for their invitation and Mikołaj Jerzykiewicz and Alosza Pamyatnykh for carefully reading the manuscript and their comments. The EC is acknowledged for the establishment of the European Helio- and Asteroseismology Network (HELAS, No. 026138), which made the authors' participation at this meeting possible.

\section{References}

Aerts, C., Thoul, A., Daszyńska, J., et al. 2003, Science, 300, 1926

Ahmad, A., \& Jeffery, C. S. 2005, A\&A, 437, L51

Ahmad, A., \& Jeffery, C. S. 2008, in "Hydrogen-Deficient Stars", eds. K. Werner and T. Rauch, ASP Conf. Ser., 391, 261

Asplund, M., Grevesse, N., Sauval, A. J., et al. 2005, A\&A, 431, 693

Barlow, B. N., Dunlap, B. H., Rosen, R., \& Clemens, J. C. 2008, ApJ, 2008, 688, L95

Basu, S., \& Antia, H. M. 2008, Phys. Rep., 457, 217

Bazot, M., Vauclair, S., Bouchy, F., \& Santos, N. C. 2005, A\&A, 440, 615

Böhm-Vitense, E. 1958, Z. Astrophys., 46, 108

Buchler, J. R., \& Szabo, R. 2007, ApJ, 660, 723

Canuto, V. M., Goldman, I., \& Mazzitelli, I. 1996, ApJ, 473, 550

Charpinet, S., Fontaine, G., Brassard, P., et al. 1997, ApJ, 483, L123

Daszyńska-Daszkiewicz, J., Dziembowski, W. A., Pamyatnykh, A. A., \& Goupil, M.-J. 2002, A\&A, 392, 151 
Daszyńska-Daszkiewicz, J., Dziembowski, W. A., \& Pamyatnykh, A. A. 2003, A\&A, 407, 999

Daszyńska-Daszkiewicz, J., Dziembowski, W. A., Pamyatnykh, A. A., et al. 2005a, A\&A, 438, 653

Daszyńska-Daszkiewicz, J., Dziembowski, W. A., \& Pamyatnykh, A. A. 2005b, A\&A, 441, 641

Daszyńska-Daszkiewicz, J., Dziembowski, W. A., \& Pamyatnykh, A. A. 2007, AcA, 57, 11

Dufour, P., Liebert, J., Fontaine, G., \& Behara, N. 2007, Nature 450, 522

Dziembowski, W. A., \& Goode, P. R. 1992, ApJ, 394, 670

Dziembowski, W. A., \& Jerzykiewicz, M. 1996, A\&A, 306, 436

Dziembowski, W. A., \& Pamyatnykh, A. A. 1991, A\&A 248, L11

Dziembowski, W. A., \& Pamyatnykh, A. A. 1993, MNRAS, 226, 204

Dziembowski, W. A., \& Pamyatnykh, A. A. 2008, MNRAS, 385, 2061

Fontaine, G., Brassard, P., Charpinet, S., et al. 2008, in "Hot Subdwarf Stars and Related Objects", eds. U. Heber, R. Napiwotzki, and C. S. Jeffery, ASP Conf. Ser., 392, 231

Fontaine, G., \& Brassard, P. 2008, PASP, 120, 104

Fontaine, G., Brassard, P., Green, B., et al. 2008a, A\&A, 486, L39

Fontaine, G., Brassard, P., \& Dufour, P. 2008b, A\&A, 483, L1

Green, E. M., Fontaine, G., Reed, M. D., et al. 2003, ApJ 583, L31

Gough D., 1977, ApJ, 214, 196

Goupil, M. J., Michel, E., Lebreton, Y., Baglin, A., 1993, A\&A, 268, 546

Goupil, M.-J., Dziembowski, W. A., Pamyatnykh, A. A., \& Talon, S. 2000, in "Delta Scuti and Related Stars", eds. M. Breger and M. H. Montgomery, ASP Conf. Ser., 210, 267

Grevesse, N., Noels, A. 1993, in "Origin and Evolution of the Elements", eds. N. Pratzo,

E. Vangioni-Flam, and M. Casse, Cambridge Univ. Press, Cambridge, p. 15

Handler, G. 2009, CoAst, 159, 42

Howarth I. D., Bolton, C. T., Crowe, R. A., et al. 1993, ApJ, 417, 338

Iben, J., Kaler, J. B., Truran, J. W., \& Renzini, A. 1983, ApJ, 264, 605

Iglesias, C. A., \& Rogers, F. J. 1991, ApJ, 371, 408

Iglesias, C. A., \& Rogers, F. J. 1996, ApJ, 464, 943

Jeffery, C. S., Drilling, J. S., Harrison, P. M., et al. 1997, A\&AS, 125, 501

Jeffery, C. S., \& Saio, H. 1999, MNRAS,308, 221

Jeffery, C. S., \& Saio, H. 2006, MNRAS,372, L48

Jeffery, C. S. 2008a, CoAst, 157, 240

Jeffery, C. S. 2008b, in "Hydrogen-Deficient Stars", eds. K. Werner and T. Rauch, ASP Conf. Ser., 391, 53

Jeffery, C. S. 2008c, IBVS, 5817, 1

Karoff, C., Arentoft, T., Glowienka, L., et al. 2008, MNRAS, 386, 1085

Kaufer, A., Stahl, O., Prinja, R. K., \& Witherick, D., 2006, A\&A, 447, 325

Keller, S. C. 2008, ApJ, 677, 483

Kochukhov, O. 2009, CoAst, 159, 61

Kołaczkowski, Z., Pigulski, A., Soszyński, I., et al. 2006, MmSAI, 77, 336

Kilkenny, D., Koen, C., O'Donoghue, D., \& Stobie, R. S. 1997, MNRAS, 285, 640

Knapp, G. R., Young, K., Lee, E., \& Jorissen, A. 1998, ApJS, 117, 209

Lenz, P., Pamyatnykh, A. A., Breger, M., \& Antoci, V. 2008, A\&A, 478, 855

Lutz, R., Schuh, S., Silvotti, R., et al. 2008, in "Hot Subdwarf Stars and Related Objects", eds.

U. Heber, C. S. Jeffery, and R. Napiwotzki, ASP Conf. Ser., 392, 339

Maeder, A., Georgy, C., \& Meynet, G. 2008, A\&A, 479, L37

Mathis, S., Talon, S., Pantillon, F.-P., \& Zahn, J.-P. 2008, SoPh, 251, 101

Meynet, G., \& Maeder, A. 2000, A\&A, 361, 101 
Maeder, A., \& Meynet, G. 2000, ARA\&A, 38, 143

Miglio, A., Montalbán, J., \& Dupret, M.-A. 2007, MNRAS, 375, L21

Miglio, A., Montalbán, J., Noels, A., \& Eggenberger P. 2008, MNRAS, 386, 1487

Montalbán, J., D'Antona, F. 2006, MNRAS, 370, 1823

Montalbán J., \& Miglio, A. 2008, CoAst, 157, 160

Montgomery, M. H., Williams, A. K., Winget, D. E., et al. 2008, ApJ, 678, L51

Moskalik, P., Buchler, J. R., \& Marom, A. 1992, ApJ, 385, 685

Moskalik, P., \& Dziembowski, W. A. 1992, A\&A, 256, L5

Moskalik, P., \& Kołaczkowski, Z. 2008a, arXiv:0807.0615

Moskalik, P., \& Kołaczkowski, Z. 2008b, arXiv:0807.0623

Moskalik, P., Kołaczkowski, Z., \& Mizerski, T. 2004, ASP Conf. Ser., 310, 498

Napiwotzki, R. 2008, in "Hydrogen-Deficient Stars", eds. K. Werner and T. Rauch, ASP Conf. Ser., 391, 257

Neilson, H. R., \& Lester, J. B. 2008, ApJ, 684, 569

Owocki, S. 2004, in "Evolution of Massive Stars, Mass Loss and Winds", eds. M. Heydari-Malayeri, Ph. Stee, and J.-P. Zahn, EAS Publ. Ser., 13, 163

Owocki, S. 2008, in "Mass Loss from Stars and the Evolution of Stellar Clusters", eds. A. de Koter, L. J. Smith, and L. B. F. M. Waters, ASP Conf. Ser., 388, 57

Pamyatnykh, A. A., \& Ziomek, W. 2007, CoAst, 150, 207

Pamyatnykh, A. A., Handler, G., \& Dziembowski, W. A. 2004, MNRAS, 350, 1022

Pigulski, A., \& Kołaczkowski, Z. 2002, A\&A, 388, 88

Quirion, P. O., Fontaine, G., \& Brassard, P. 2007, CoAst, 150, 247

Rodriguez-Lopez, C., Ulla, A., \& Garrido, R. 2007, MNRAS, 379, 1123

Saio, H. 1993, MNRAS, 260, 465

Saio, H., \& Jeffery, C. S. 1988, ApJ, 328, 714

Lee, U., \& Saio, H. 1997, ApJ., 491, 839

Savonije, G. J. 2007, A\&A, 469, 1057

Seaton, M. J. 1992, RMxAA, 23, 180

Seaton, M. J. 1996, MNRAS, 279, 95

Seaton, M. J. 2007, MNRAS, 382, 245

Schuh, S., Huber, J., Dreizler, S., et al. 2006, A\&A, 445, L31

Silvotti, R., Schuh, S., Janulis, R., et al. 2007, Nature 449, 189

Simon, R. N. 1982, ApJ, 260, L87

Smolec, R., \& Moskalik, P. 2008a, AcA, 58, 233

Smolec, R., \& Moskalik, P. 2008b, AcA, 58, 193

Soufi, F., Goupil, M-J., \& Dziembowski, W. A. 1998, A\&A, 334, 911

Soszyński, I., Poleski, R., Udalski, A., et al. 2008a, AcA, 58, 153

Soszyński, I., Poleski, R., Udalski, A., et al. 2008b, AcA, 58, 163

Stein, R. F., \& Nordlund, A. 1998, ApJ, 499, 914

Steffen, M. 2007, in "Convection in Astrophysics", eds. F. Kupka, I. Roxburgh, and K. Chan, IAU Symposium, 239, 36

Talon, S., \& Charbonnel, C. 2005, A\&A, 440, 981

Théado, S., Dupret, M.-A., Noels, A., \& Ferguson, J. W. 2009, A\&A 493, 159

Townsend, R. 1997, MNRAS, 284, 839

Townsend, R. 2003, MNRAS, 343, 125

Townsend, R. 2007, in "Unsolved Problems in Stellar Physics", AIP Conf. Proceedings, 948, 345 
Townsend, R., \& MacDonald, J. 2008, in "Massive Stars as Cosmic Engines", eds. F. Bresolin, P. A. Crowther, and J. Puls, IAU Symposium, 250, 161

Udalski, A., Soszyński, I., Szymański, M., et al. 1999, AcA, 49, 45

Unno, W., 1967, PASJ, 19, 140

Webbink, R. F. 1984, ApJ, 277, 355

Winget, D. E. 1998, J. Phys., Condensed Matter, 10, 11247

Wood, P. R. 2007, in "From Stars to Galaxies: Building the Pieces to Build Up the Universe", eds. A. Vallenari, R. Tantalo, L. Portinari, and A. Moretti, ASP Conf. Ser., 374, 47

Woudt, P. A., Kilkenny, D., Zietsman, E., et al. 2006, MNRAS, 371, 1397

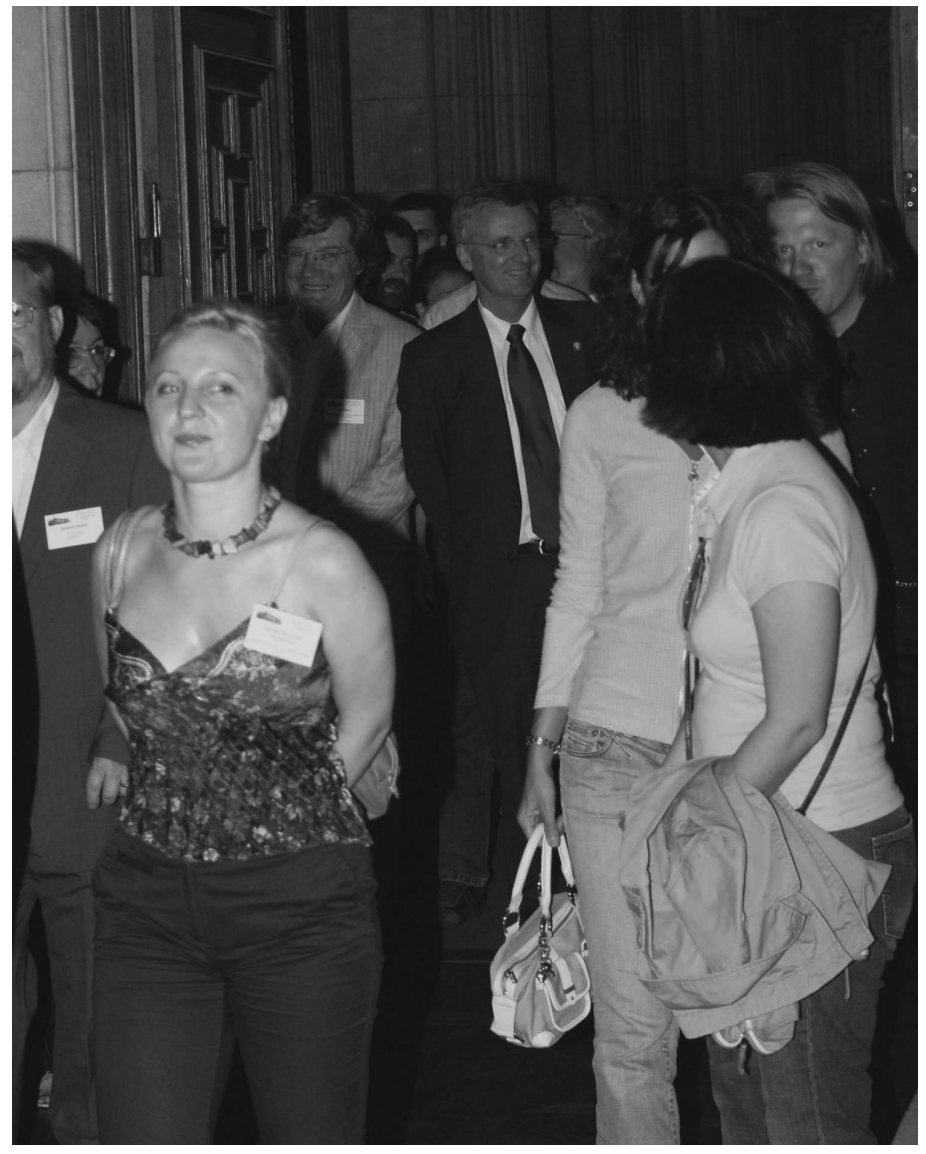

